\title{
Tackling Non-linearity in Cavity Perturbation using Machine Learning Approach
}

\author{
Zubair Akhter, and Atif Shamim \\ CEMSE, Division, KAUST \\ Jeddah, Saudi Arabia \\ line 5: zubair.zakhter@kaust.edu.sa:
}

\author{
Ahmad Khusro \\ Dept. of Electrical Engineering \\ Jamia Millia Islamia (A Central Univ.) \\ New Delhi, India
}

\author{
Abhishek K. Jha \\ Dept. of Electrical Engineering \\ Indian Institute of Technology Tirupati \\ Andhra Pradesh, India
}

\begin{abstract}
This paper presents a unique design of a cylindrical cavity to identify the dielectric materials with high resolution using the supervised machine learning algorithm. The mountable design of an aluminum-based cylindrical cavity records a quality factor of more than 9000 and provides an easy assembly of samples to be tested. The linear region of the cavity precisely provides the identification of dielectric samples in the range of 1 to 20 within $99 \%$ of accuracy using the standard cavity formulation. On the other hand, the proposed machine learning approach works effectively in the non-linear region of the cavity and predicts the dielectric properties accurately in the wide range dielectric constant starting from 20-45 with a typical error of $0.35 \%$. The non-linearity of the cavity output is modeled using the cascade feedforward architecture of Artificial Neural Network (ANN) for multiinput variables extracted from the simulations. The model is trained using a well-known Bayesian regularization algorithm with an adequate number of samples and subsequently tested over a large sample of novel test input. The mean square error of test samples in the range of $10^{-4}$ and correlation coefficient (R) near 1 demonstrates the effectiveness of the approach in dielectric testing using the proposed cavity.
\end{abstract}

Keywords-cylindrical cavity, dielectric testing, machine learning algorithm, nonlinearity, resonant technique.

\section{INTRODUCTION}

The identification of various dielectric materials at sub-6 $\mathrm{GHz}$ frequency with higher resolution is essential for the $5 \mathrm{G}$ technology. Exploiting the interaction between materials and EM energy, a large number of microwave sensors based on resonant and transmission-reflection approaches have been developed [1]. Moreover, microwave sensors are attractive and growing rapidly for various industrial sensing applications, such as material identification [2]-[3], displacement detection [4]-[5], and biomedical sensing [6][7] due to their high measurement accuracy, fast detection ability, low-cost, and noninvasive characteristics. Particularly, resonant methods, in which the material under test (MUT) interacts with a resonant structure, are highly used due to the accuracy and straightforward analysis [8][11]. However, as discussed in [8], the resonant cavity technique has a series of limitations mostly arising from nonlinearity. It is shown that the non-linearity in the cavity perturbation technique arises when $V_{S} / V_{C}>0.005$, where $V_{s}$ and $V_{c}$ are the volumes of the sample under test and the cavity. The relaxation in limitations as suggested in [8]-[9] are applicable when the resonant frequency and quality factor can be directly measured from the scattering coefficients. Recently, computationally intelligent sensors using the machine learning (ML) techniques and in particular Artificial Neural Network (ANN) [12] are gaining popularity in frequency-dependent dielectric constant estimation [13], which can further accelerate the materials property predictions at the microwave frequency [14].

The novelty of this paper is twofold, first, a low-profile, high quality, aluminum-based, cylindrical cavity with mountable design is presented in detail, followed by the introduction of the ML technique to exempt the error-prone measurement in the non-linear region of the cavity. The proposed model implicitly maps the multi-input variable such as quality factor $(Q)$, center or resonant frequency, 3-dB bandwidth, measured S-parameters, and measured frequency in higher dimensional space to predict the dielectric property of the cavity. The proposed approach uses over 0.5 million frequency data points/samples for training and more than 0.35 million frequency data points/samples of novel samples to test the effectiveness of the approach. The proposed technique provides an excellent match with the input data and can be used for the identification of microwave materials in the industry.

\section{DESIGN OF MicrowaVE CIRCUlar CAVITY}

The CST Microwave Studio is used to model the cylindrical resonant cavity as shown in Fig. 1. The cavity is made of aluminum and design to operate at $5 \mathrm{GHz}$. The cavity is fed with two coaxial SMA-type ports as shown in the inset of Fig. 1. The cavity is made up of two parts i.e., top and bottom, which can be easily fixed or detached from each other. Such mountable arrangement of the cavity design helps while placing the test sample inside the cavity for dielectric testing. 


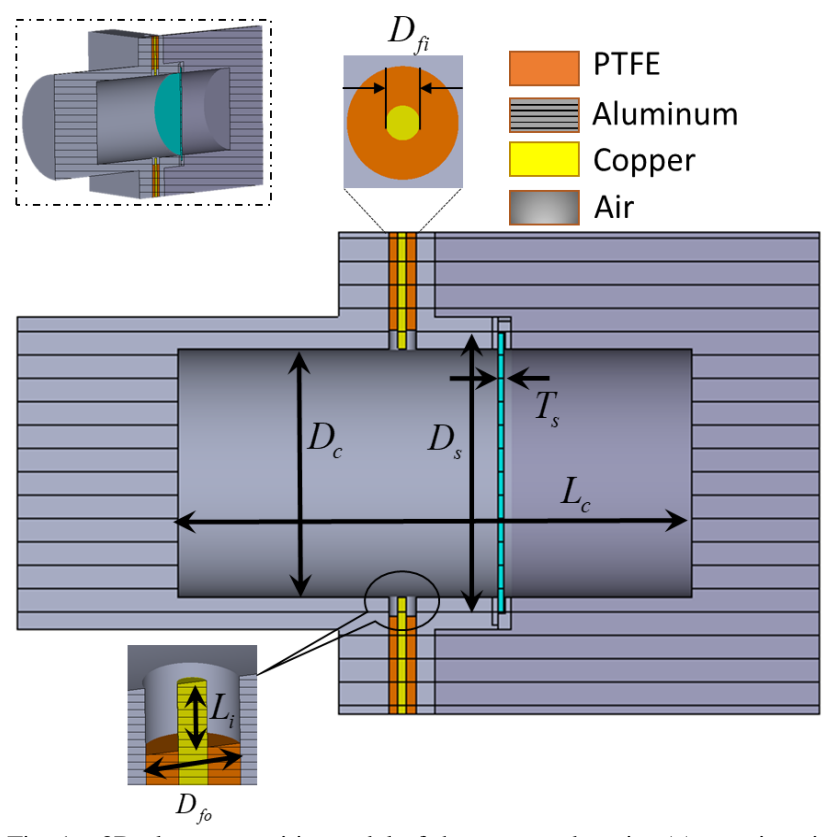

Fig. 1. 3D electromagnitic model of the proposed cavity (a) exterior view and (b) cut view.

The design parameters of the cavity are listed in Table-I. The model is simulated with the frequency domain solver of CST for evaluating the scattering parameters of the cavity. Fig. 2 shows the simulated transmission coefficient $\left(S_{21}\right)$ for the designed cavity, where the resonant frequency and quality factor are found to be $f_{\mathrm{r}}=5 \mathrm{GHz}$ and $Q_{0}=9231$ respectively. It is to be noted here that the higher quality factor is desirable for measuring low-loss dielectric samples. To check the sensitivity of the designed cavity, the dielectric properties of the test sample are varied and corresponding changes in the cavity resonant frequency are observed as shown in Fig. 3. In a different simulation study, the loss tangent data of the test sample is varied and the behavior of the transmission coefficient is analyzed as shown in Fig. 4.

TABLE I. CAVITY DESIGN PARAMETER

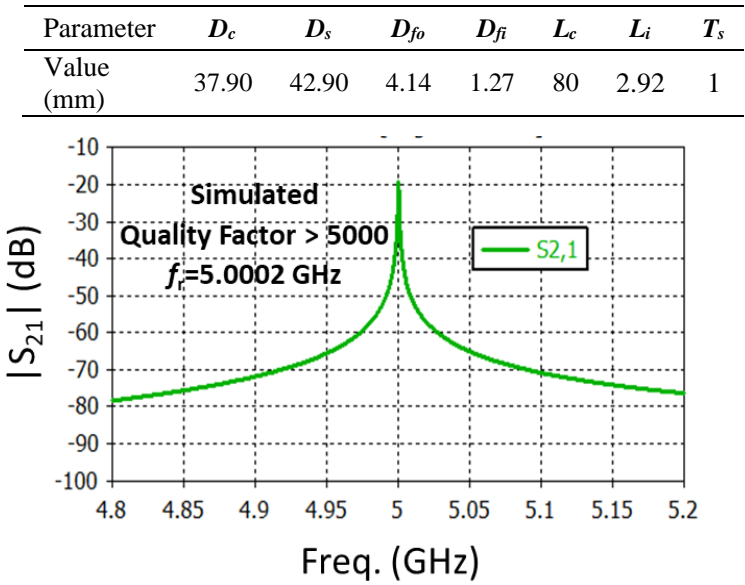

Fig. 2. Simulated transmission coefficient $\left(S_{21}\right)$ of the proposed cavity.

In Fig. 5, the change in the resonant frequency w.r.t. changes in the dielectric permittivity of the test sample arebeing plotted. As can be seen here, this relationship is linear for lower permittivity values $<20$, however, for higher values of permittivity, the transfer characteristic is no longer following a linear relationship. This is a typical problem with the conventional cavity perturbation reconstruction that usually being referred to as overloading of the cavity. In this paper, it is tried to tackle this non-linearity with the help of the supervised machine learning presented in the next section.

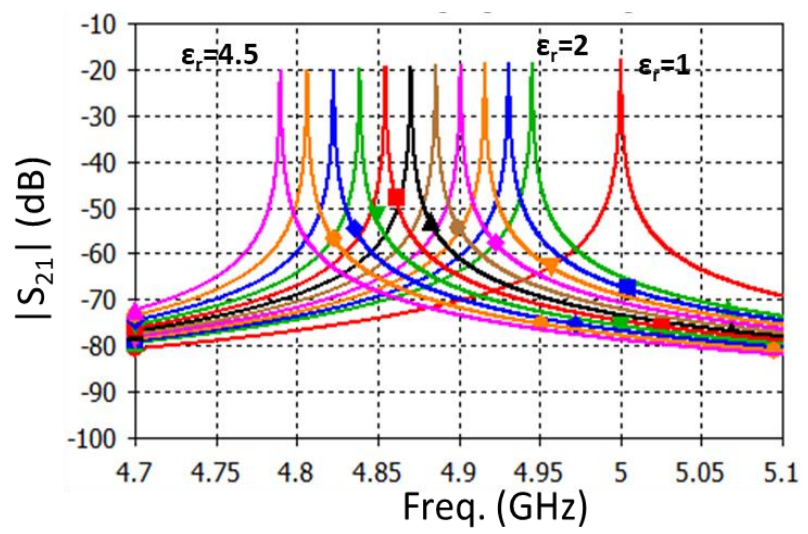

Fig. 3. Resonant frequency $\left(f_{\mathrm{r}}\right)$ vs dielectric permittivity of the test sample.

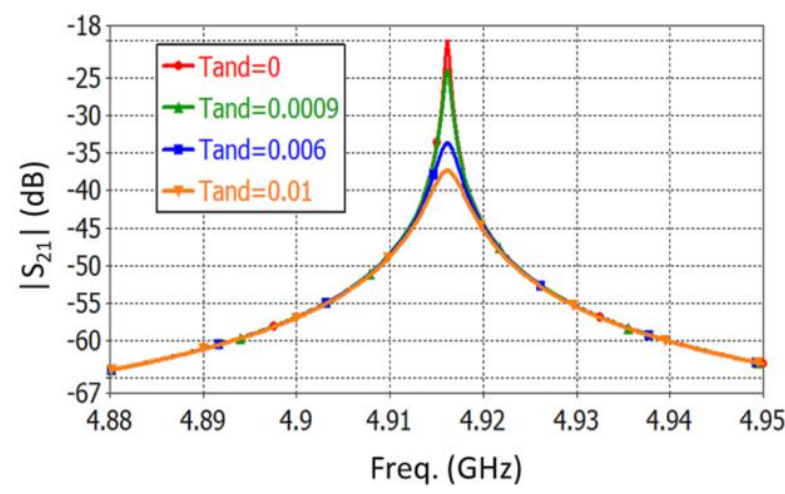

Fig. 4. Simulated transmission coefficient $\left(S_{21}\right)$ for various loss tangents $\left(\varepsilon_{\mathrm{r}}=2.5\right)$.

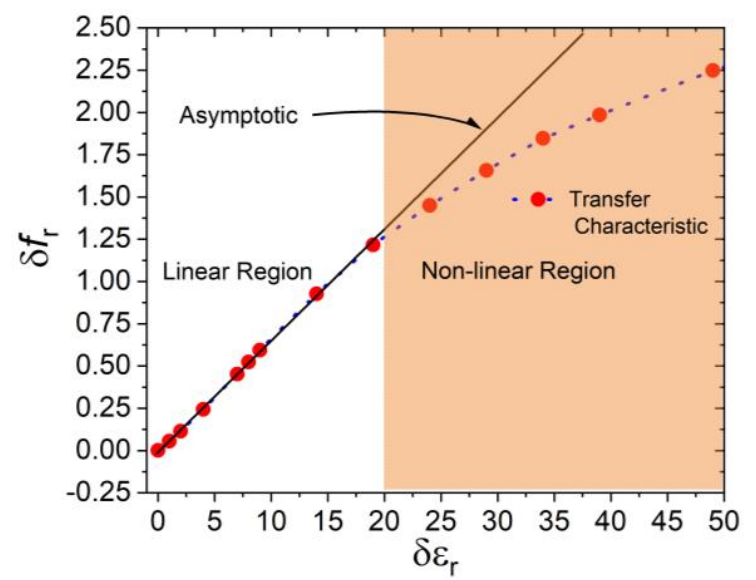

Fig. 5. Charge in the resonant frequency $\left(f_{\mathrm{r}}\right)$ of the cavity vs change in the dielectric permittivity $\left(\varepsilon_{\mathrm{r}}\right)$ of the test sample.

\section{PROPOSED ANN FRAMEWORK FOR THE DETERMINATION OF COMPLEX PERMITTIVITY.}

A neural network is composed of the input layer, the internal hidden layers, and the output layer [15]. In general, a neural network consists of neurons that act as a node. Multiple layers are stacked respectively in a network to form a complete structure and the neurons of respective layers are connected through synaptic weights as shown in Fig. 6. The neurons stacked in a distinct layered structure 
perform a biased weighted sum of their inputs and subsequently pass it through a linear or non-linear transfer function as per the requirement of a problem.

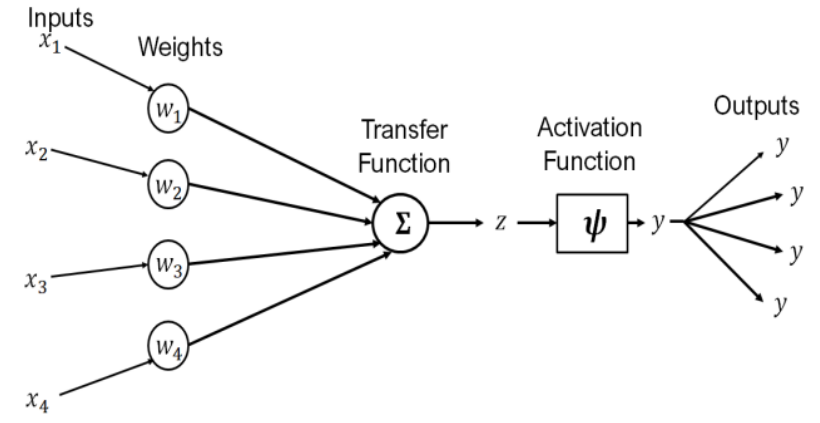

Fig. 6. Basic Artificial Neural Network model (ANN) for a single neuron

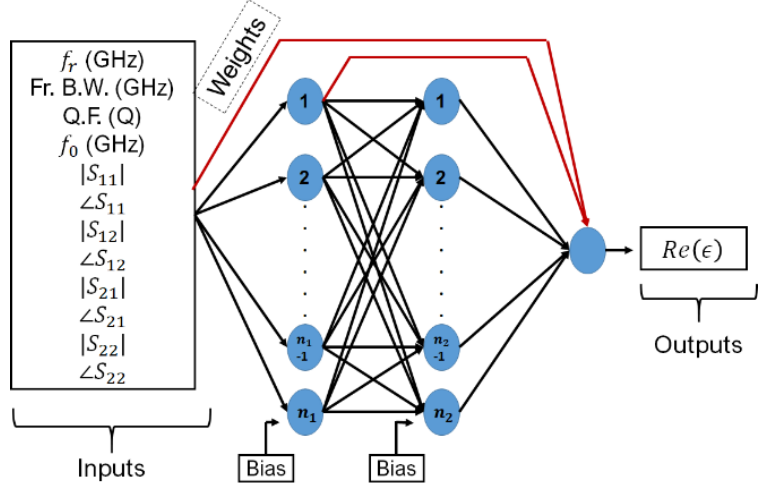

Fig. 7. Proposed ANN model for the determination of complex permittivity.

The determination of complex permittivity of the dielectric sample requires implicit mapping of 12 predictor variables into feature space as shown in Fig. 6. The prosed model maps two-port $S$-parameters and other extracted parameters such as measured unloaded resonant frequency $f_{0}$ $(\mathrm{GHz})$, loaded resonant center frequency $f_{c}$, the quality factor, 3 - $\mathrm{dB}$ bandwidth from the measurement dataset to highdimensional feature space. The proposed model uses the cascade feedforward network architecture of neural networks. and two hidden layers (with 25 neurons in each layer). The neural model for determining the complex permittivity is developed using 5,50,000 samples of which $60 \%$ of data is utilized for training and the rest $40 \%$ is used for testing and validation. At a preprocessing stage, the response of the model is divided by 100 in the manner that it adjusts in the range of $[0,1]$. This is done to expedite the convergence of the model at the training stage. The response of the first, second, and third layers is passed through the tansigmoid activation function to model such complex behavior. The neural model prefers the Nguyen-Widrow method for the initialization of weights and bias [16]. The method aids in the uniform and even distribution of neurons over the input space and therefore helps in expediting the convergence and training process. The model is trained using a well-known Bayesian algorithm to determine the optimal sets of weight and bias. The Bayesian algorithm ensures better generalization capability for the model. The termination criterion for the developed ML algorithm is either achieving the desired training mean square error (MSE) of 1e-4 or reaching the iteration mark of 250 . The weights and bias are adjusted in a manner to find the minimum value of fitness function (FF) expressed using the MSE and correlation coefficient (R) as given in (1) and (2), respectively. It is quite evident from Table-II that the proposed model provides very good MSE for the training as well as test set and is highly effective in the determination of real part of complex permittivity in linear as well as non-linear regions specified in Fig. 5. The correlation coefficient plot (R) between measured and predicted values of permittivity for complete test and train set samples are found to be 0.9956 and 0.9958 respectively which demonstrates the excellent prediction ability of the model. On the other hand, the relative error between the measured and modeled value is plotted as a function of measured permittivity as shown in Fig. 8.

$$
\mathrm{MSE}=\frac{1}{N} \sum_{i=1}^{N}\left(y_{m}-y_{p}\right)^{2}
$$

$$
R=\sqrt{\frac{\left(N \sum_{i=1}^{N} y_{m} y_{p}-\sum_{i=1}^{N} y_{p} \sum_{i=1}^{N} y m\right)^{2}}{\left(N \sum_{i=1}^{m} y_{p}^{2}-\left(\sum_{i=1}^{N} y_{p}\right)^{2}\right)\left(N \sum_{i=1}^{N} y_{m}^{2}-\left(\sum_{i=1}^{N} y_{p}\right)^{2}\right)}} \in[0,1]
$$

TABLE II. MOdELEd VAlues Of Real PART OF COMPLEX PERMITTIVITY

\begin{tabular}{|c|c|c|l|}
\hline \multicolumn{2}{|c|}{ Train Data Set } & \multicolumn{2}{c|}{ Test Data Set } \\
\hline True Value & Predicted & True Value & Predicted \\
\hline 10.5 & 10.510526 & 2.3 & 2.298197088 \\
\hline 14.3 & 14.3005641 & 3.7 & 3.700111645 \\
\hline 16.6 & 16.5988914 & 4.8 & 4.804281987 \\
\hline 4.9 & 4.90789166 & 9.0 & 9.006386621 \\
\hline 7.0 & 7.00216325 & 13.5 & 13.48289906 \\
\hline 3.4 & 3.39796138 & 14.4 & 14.4623516 \\
\hline 18.1 & 18.0871981 & 15.9 & 15.89338377 \\
\hline 18.8 & 18.8000755 & 16.5 & 16.51527447 \\
\hline 19.2 & 19.1998021 & 17.9 & 17.87462377 \\
\hline 2.0 & 1.99394668 & 18.4 & 18.39064568 \\
\hline 17.6 & 17.59928973 & 20 & 19.96001278 \\
\hline 19.8 & 19.7790501 & 35 & 35.077012 \\
\hline 25 & 24.9692 & 45 & 45.1019876 \\
\hline
\end{tabular}

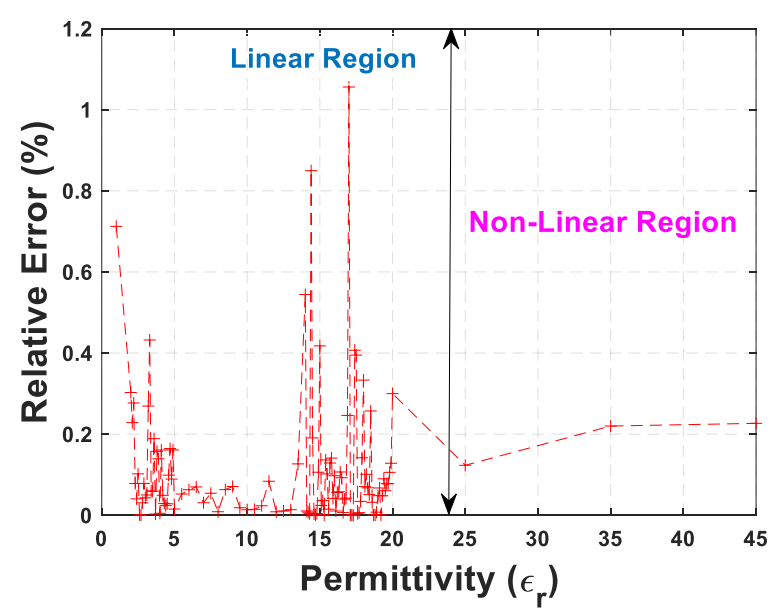

Fig. 8. Relative error between the measured and predicted values of permittivity plotted as a function of measured permittivity.

\section{CONCLUSION}

In this paper, a cylindrical cavity for the measurement of the dielectric properties at the $5 \mathrm{G}$ band is designed. The proposed cavity is made up of two detachable parts for ease in sample placement and shows a very high-quality factor $Q_{0}$ 
$=9231$. To deal with the non-linearity caused due to the overloading of the cavity, an artificial neural network-based model is developed. This model is trained on huge data set of about 0.3 million data points and suitably tested with the remaining 0.2 million data points. Based on the observed performance, it is found that the proposed model can predict the dielectric properties of a test sample (relative permittivity and loss tangent) within a $1 \%$ error.

\section{REFERENCES}

[1] L. Chen, et al., Microwave Electronics: Measurement and Materials Characterization, Chichester, U.K. Wiley, 2004, pp. 142-186.

[2] Y. J. Mao, Y. J. Zhang, Z. R. Chen, and M. S. Tong, "A noncontact microwave sensor based on cylindrical resonator for detecting concentration of liquid solutions," IEEE Sensors J., vol. 21, no. 2, pp. 1208-1214, Jan. 2021.

[3] S. Deif and M. Daneshmand, "Long array of microwave sensors for realtime coating defect detection," IEEE Trans. Microw. Theory Tech., vol. 68, no. 7, pp. 2856-2866, Jul. 2020.

[4] T. Cheng, C. H. Chio, K. W. Tam, and P. Y. Lau, "An angular displacement microwave sensor with $360^{\circ}$ dynamic range using multimode resonator," IEEE Sensors J., vol. 21, no.3, pp. 2899-2907, Feb. 2021.

[5] A. K. Jha, A. Lamecki, M. Mrozowski, and M. Bozzi, "A microwave sensor with operating band selection to detect rotation and proximity in the rapid prototyping industry," IEEE Trans. Industrial Electron., vol. 68, no. 1, pp. 683-693, Jan. 2020.

[6] J. A. Osterberg, N. Dahal, R. Divan, C. S. Miller, D. Moline, T. P. Caldwell, X. Yu, S. W. Harcum, and P. Wang, "Microwave sensing of yeast cell species and viability," IEEE Trans. Microw. Theory Tech., vol. 69, no. 3, pp. 1875-1886, Mar. 2021.

[7] M. C. Cebedio, L. A. Rabioglio, I. E. Gelosi, R. A. Ribas, A. J. Uriz, and J. C. Moreira, "Analysis and design of a microwave coplanar sensor for non-invasive blood glucose measurements," IEEE Sensors J., vol. 20, no. 18, pp. 10572-10581, Sep. 2020.
[8] A. K. Jha and M. J. Akhtar, "A Generalized Rectangular Cavity Approach for Determination of Complex Permittivity of Materials," IEEE Trans. Instrum. Meas., vol. 63, no. 11, pp. 2632-2641, Nov. 2014.

[9] A. K. Jha, N. K. Tiwari and M. J. Akhtar, "Accurate Microwave Cavity Sensing Technique for Dielectric Testing of Arbitrary Length Samples," IEEE Trans. Instrum. Meas., vol. 70, pp. 1-10, 2021, Art no. 6006910

[10] N. K. Tiwari, A. K. Jha, S. P. Singh, Z. Akhter, P. K. Varshney and M. J. Akhtar, "Generalized Multimode SIW Cavity-Based Sensor for Retrieval of Complex Permittivity of Materials," IEEE Trans. Microw. Theory Tech., vol. 66, no. 6, pp. 3063-3072, June 2018.

[11] Salski, Bartlomiej, Jerzy Cuper, Tomasz Karpisz, Pawel Kopyt, and Jerzy Krupka. "Complex permittivity of common dielectrics in 20$110 \mathrm{GHz}$ frequency range measured with a Fabry-Pérot open resonator." App. Phys. Lett, vol.119, no. 5, 2021, 052902.

[12] S. Panda, N. K. Tiwari and M. J. Akhtar, "Computationally intelligent sensor system for microwave characterization of dielectric sheets," IEEE Sensors J., vol. 16, no. 20, pp. 7483-7493, 2016.

[13] Chen, Lihua, et al. "Frequency-dependent dielectric constant prediction of polymers using machine learning." npj Computational Materials, vol. 6, no. 1, pp. 1-9, 2020.

[14] G. Pilania, C. Wang, X. Jiang, S. Rajasekaran, and R. Ramprasad "Accelerating materials property predictions using machine learning. Scientific reports, vol. 3, no. 1, pp. 1-6, 2013.

[15] A. Khusro, et. al., "Small signal behavioral modeling technique of GaN high electron mobility transistor using artificial neural network: Anaccurate, fast, and reliable approach,"Int. Journal of $R F$ and Microwave Computer-Aided Engineering., vol. 30, no. 4, pp. 1-15, Apr. 2020.

[16] D. Nguyen and B. Widrow, "Improving the learning speed of 2-layer neural networks by choosing initial values of the adaptive weights,"IJCNN Int. Joint Conference on Neural Networks, pp. 21-26 vol.3, 1990. 